\title{
Adaptive Linear Discriminant Analysis Algorithm Applied to Motion Signal Classification in EEG Processing
}

\author{
Rui $\mathrm{Xu}^{1, \mathrm{a}}$, Haoyue Tang ${ }^{2, b}$ \\ ${ }^{1}$ School of Computer Science and Engineering, University of Electronic Science and Technology of \\ China, Chengdu 611731, China \\ ${ }^{2}$ School of Computer Science and Engineering, University of Electronic Science and Technology of \\ China, Chengdu 611731, China \\ axurui.stone@foxmail.com, btang.haoyue@foxmail.com
}

Keywords: EEG, brain computer interface, aCSP algorithm, aLDA algorithm, LDA algorithm, updating coefficient.

\begin{abstract}
In view of the current development research on electroencephalograph (EEG) brain computer interface (BCI), which shall not be limited to use linear discriminant analysis (LDA) algorithm only. The brain computer interface that uses multi channel and multi type EEG signal is gradually fusing on other ways that can better reflect the brain activity. Based on the EEG computer interface technology, we can use adaptive linear discriminant analysis (aLDA) algorithm and human-computer interaction method, also regulate adaptive updating coefficient (UC) to reflect the brain thinking activity better. In this paper, we use adaptive common spatial pattern (aCSP) algorithm to extract feature and better classification algorithm to process the EEG, which can improve the efficiency, accuracy and stability of signal classification.
\end{abstract}

\section{Introduction}

Feature recognition rate on EEG is a core part of brain information classification, because pattern recognition of EEG result shall directly influence the control effect of multi-modal interface [1]. In the part of feature extraction and pattern recognition, using the heavy disability's EEG as the control signal has encountered a problem of parameter dimension, which is insufficient and unable to control

accurately. So on the basis of heavy disability's EEG feature, finding the way of better feature parameter extraction to control more appropriately, quickly and accurately is indeed needful [2]. Most of the previous studies on the EEG are for normal people, using the time and frequency domain, parameter model and other methods to acquire feature signal extraction [3]. In addition, when in the different situation, we can regulate UC to get better recognition rate. We use linear discriminant analysis algorithm and adaptive linear discriminant analysis algorithm to process the signal, compare the result and regulate UC to get better result. As we all know EEG has the real-time, large information capacity and flexibility advantages, but it is very susceptible to be influenced by the external environment and also the signal filtering denoising links are very complex [4]. Therefore, choosing the appropriate classification algorithm is very important. To deal with this, use the adaptive way to process the EEG and we find that UC also has a great impact on the classification in the experiment.

\section{Denoising Processing and Feature Extraction}

Use the fourth BCI competition data BCICIV_calib_ds1b, BCICIV_calib_ds1c, BCICIV _calib_ds1e and BCICIV_calib_ds1g to preprocess, but the competition only provides the signal that be processed through the digital analog conversion [5]. As for this, we choose the channel T7 to do filter processing. The reason of using channel T7 was introduced in [6]. Due to the EEG contains too many noises, such as the $50 / 60 \mathrm{~Hz}$ power interference artifact, the eye blink artifact, the eye 
movement artifact etc [6]. It finds that the motor imagery signal frequency mainly contains between 3 $\sim 45 \mathrm{~Hz}$

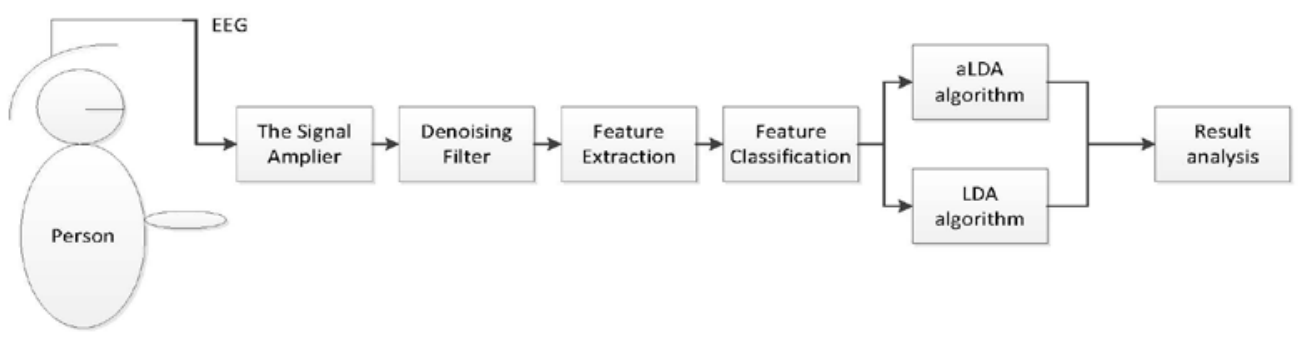

Fig. 1: EEG signal processing flow

in the experiment, so in order to prevent non-working band signal interference frequency, we need to use the spatial filtering to reduce space fuzzy influence and aCSP algorithm to extract feature, which is widely used in the BCI system based on the motor imagery. For processing two classification of motor imagery problem and this algorithm has a good effect [7]. From a view of mathematical point, the process of aCSP algorithm is the process of solving an optimization problem [8].

\section{Signal Classification Algorithm}

Time-varying EEG Signal Characteristics. Brain activity status will be influenced by external environment, such as sound, light and physical condition. The impact of factor such as emotion, attention and constant change make the EEG activity have obvious time-varying characteristic [9]. We can see from the Fig.2 to see the time-varying characteristics.

Linear Discriminant Analysis. LDA algorithm using training sample for learning to get corresponding linear classification [8]. For example, suppose there is two sample set of $Y_{1}, Y_{2}$. The number of $Y_{1}$ and $Y_{2}$ is $\mathrm{n}$. For each sample data has a $C^{*} N$ Real matrix, $C$ is said the signal channel, $N$ is said the sampling point. The classification training algorithm step [11] is in the following:

$$
\begin{aligned}
& \omega=\mathrm{A}^{-1}\left(m_{1}-m_{2}\right) \\
& \mathrm{b}=-\omega^{T} * \frac{1}{2} *\left(m_{1}+m_{2}\right) \\
& \mathrm{D}(y)=b+\omega^{T} * y
\end{aligned}
$$

In this, $A=A 1+A 2, A_{i}$ is the $i$ class sample covariance matrix. $m_{i}$ represents the $i$ mean sample. $\mathrm{i}=1,2$. $\mathrm{w}, \mathrm{b}$ are the classification parameters. For a sample to be classed the feature vector is $\mathrm{y}$. when $\mathrm{D}(\mathrm{y})>0, \mathrm{y} \in Y_{2}$. when $\mathrm{D}(\mathrm{y})<0, \mathrm{y} \in Y_{1}$.

Adaptive Linear Discriminant Analysis. Adaptive LDA algorithm uses the new input data to estimate the classification parameter to adapt to the current characteristic of data and improve the rate of correcting classification [10]. Classification of device parameter depends on the covariance $A$ and meaning vector $m_{1}, m_{2}$, so this algorithm is essentially based on the meaning vector and the covariance.

The Estimation of Meaning Vector. For the known $N$ samples in the $\operatorname{set}\{y(i)\}$, the mean $m$ is according to the following formulation definition:

$$
m=\frac{1}{N} \sum_{i=1}^{N} y(i)
$$

In the case of time variation, we can use the sliding window method to estimate the mean [11]: 


$$
m(t)=\frac{1}{\sum_{i=0}^{n-1} \omega_{i}} \sum_{i=0}^{n-1} \omega_{i} * y(t-i)
$$

$n$ is the sliding window width, $w_{i}$ is the weight coefficient. Let the estimated mean value at time $t-1$ replace the last $n-1$ sampling data, we can conclude this formula:

$$
m(t)=(1-U C) * m(t-1)+U C * y(t)
$$

In this, the $y(t)$ is the input of moment $t, U C$ is the updating adaptive coefficient.

Covariance Estimation. As for the $N$ sample set $\{y(i)\}$, its covariance matrix $A$ is according to the following formula definition:

$$
A=\frac{1}{N} \sum_{i=1}^{N}[y(i)-m]^{T} *[y(i)-m]
$$

In the case of time variant, estimation of covariance of $t$ time for $A(t)$ is:

$$
A(t)=(1-U C) A(t-1)+U C[1-y(t)]^{T}[1-y(t)]
$$

$y(t)$ represents the current input sample, $U C$ is the update coefficient. In order to simplify the calculation, through the transformation, estimate the $A(t)^{-1}, A(t-1)^{-1}$ and get the recursion formula :

$$
A(t)^{-1}=\frac{1}{1-U C}\left[A(t-1)^{-1}-\frac{1}{\frac{1-U C}{U C}+y(t)^{T} g(t)} g(t) g(t)^{T}\right]
$$

In this, $g(t)=A(t-1)^{-1} y(t)$. On the mean vector and the covariance matrix, we do the online adaptive estimation, so we can complete the re-estimation of classification parameter and get the recursion formula [11]:

$$
\begin{aligned}
& A(t)^{-1}=\frac{1}{1-U C}\left[A(t-1)^{-1}-\frac{1}{\frac{1-U C}{U C}+y(t)^{T} g(t)} g(t) g(t)^{T}\right] \\
& \omega(t)=A(t)^{-1}\left[m_{2}(t)-m_{1}(t)\right] \\
& b(t)=-\omega(t)^{T} \frac{1}{2}\left[m_{2}(t)+m_{1}(t)\right] \\
& c_{t}=F\{D[y(t)]\}=F\left[b(t-1)+\omega(t-1)^{T} y(t)\right] \\
& m_{c_{t}}(t)=(1-U C) * m_{c_{t}}(t-1)+U C * y(t)
\end{aligned}
$$

In this, $c_{t}$ represents the category labelling, $c_{t} \in\{1,2\} . F\{D[y(t)]\}$ represents sample $y$ kind category judgment function.

Algorithm Evaluation. As we can see the data above, it can conclude that in some situation when we use the aLDA algorithm to process the signal parameter, it can improve the correct rate of recognition. The initial parameter of algorithm classification is made by training the sample generation, but it will adjust according to the experiment sample [12]. From the TABLE1, we can see 
that aLDA algorithm is generally higher than LDA algorithm in the average correct classification rate. But in some case, the correct rate aLDA of algorithm has decreased, because that aLDA is an unsupervised adaptive algorithm, a new input sample category depends on the moment of the last classification [11].

\section{Experiment Result and Discussion}

See from Fig.2, use the fourth BCI competition data to process, the brain computer signal that not through processed has a lot of clutter waveform [13]. We need to take the spatial filter to process the signal to get the signal we want. After filter denoising, project the source signal onto a two-dimension feature space [14]. See from Fig.3 that four different EEG signals in a two-dimensional feature space, left and right hand signal are mostly clustered together. Set UC as 0.05 and through the aCSP feature extraction algorithm and get the further classification result we need. After LDA and aLDA algorithm processing, we can see the result in Fig.4 and Fig.5. In some situation, the LDA algorithm has a good result, because the LDA algorithm set up the threshold for classification based on the sample mean and variance, aLDA algorithm will adjust according to the sample [15]. In Fig.7, we adjust the coefficient UC and calculate the corresponding correct rate. We find that when the result of LDA algorithm is good, such as subject (1) and subject (2) and no matter how we regulate UC, the result would not like the result of LDA algorithm. But in the subject (3) and subject (4), when the result of LDA algorithm is poor, we can regulate UC to get better classification result. Also from Fig.7, the overall classification correct rate is on the decline.

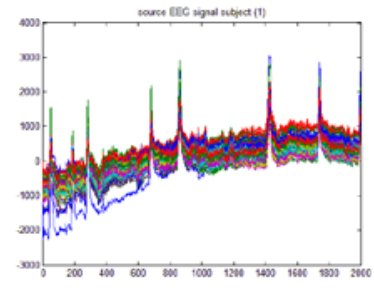

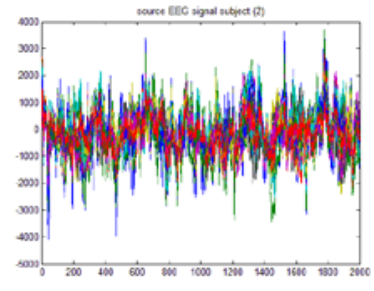

(1) (2)

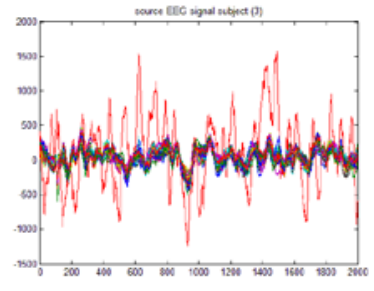

(3)

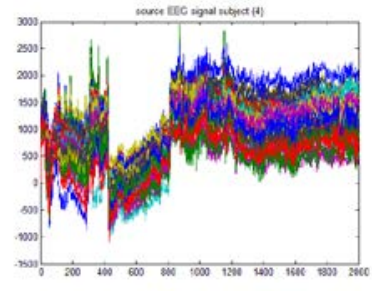

(4)

Fig. 2: The EEG signal without processing

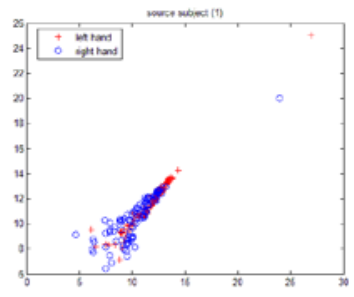

(1)

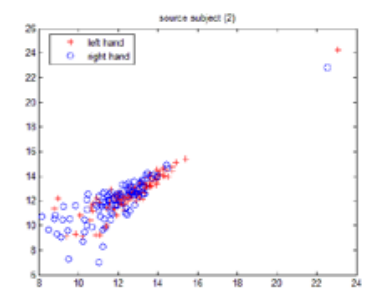

(2)

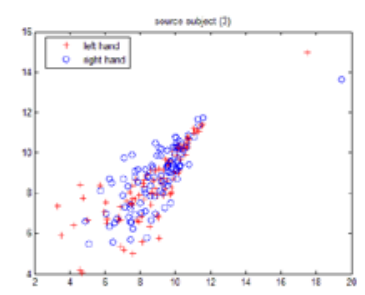

(3)

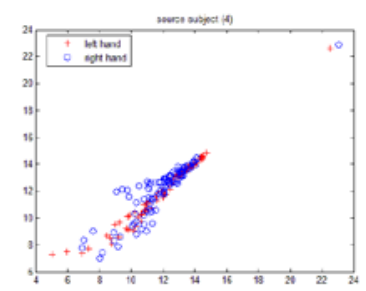

(4)

Fig. 3: Projection signal in two-dimensional feature space

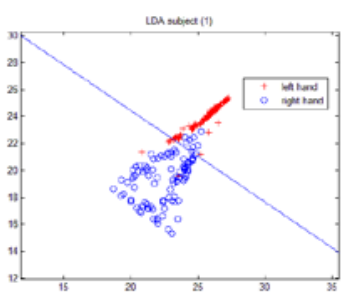

(1)

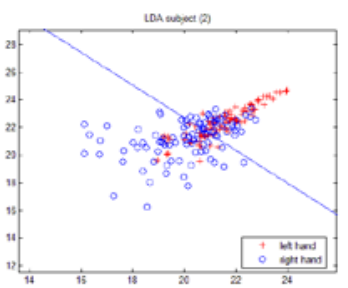

(2)

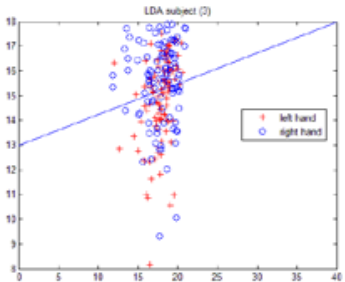

(3)

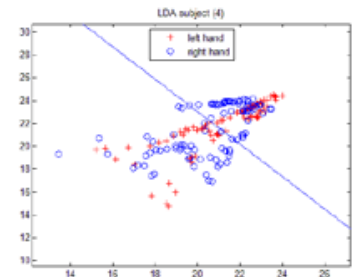

(4)

Fig. 4: The result of using aCSP algorithm and LDA algorithm, UC=0.05 


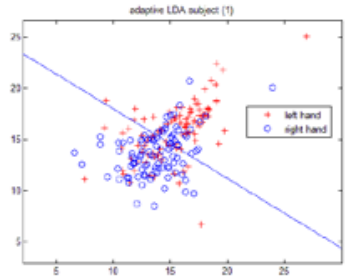

(1)

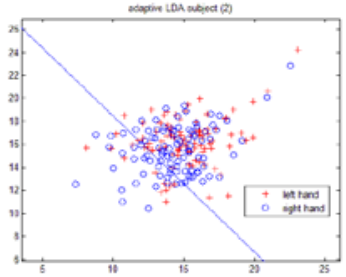

(2)

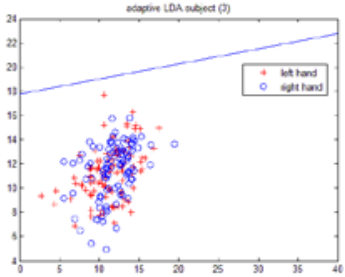

(3)

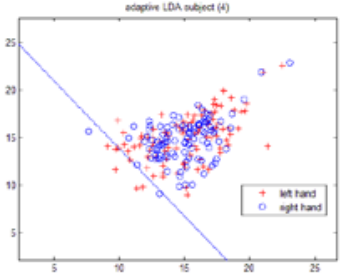

(4)

Fig. 5: The result of using aCSP algorithm and aLDA algorithm, $U C=0.05$, The blue line is the last updating line of the aLDA algorithm in the 100 times updating

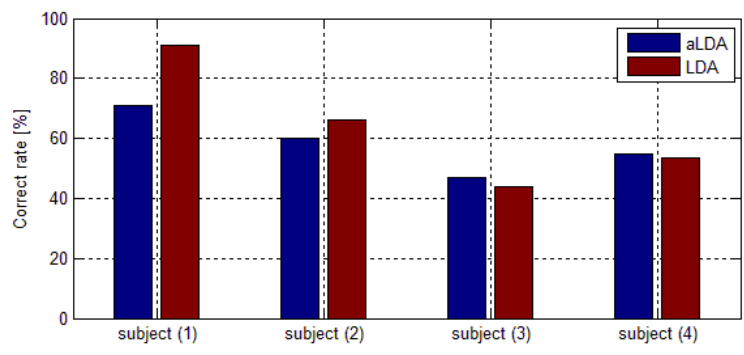

Fig. 6: The result of aLDA and LDA algorithm, UC=0.05

Table 1: The correct rate of aLDA and LDA algorithm, UC=0.05

\begin{tabular}{lcccc}
\hline Classifier & Subject (1)[\%] & Subject (2)[\%] & Subject (3)[\%] & Subject (4)[\%] \\
\hline aLDA & 71.00 & 60.00 & 47.00 & 55.00 \\
LDA & 91.00 & 66.00 & 44.00 & 53.50
\end{tabular}

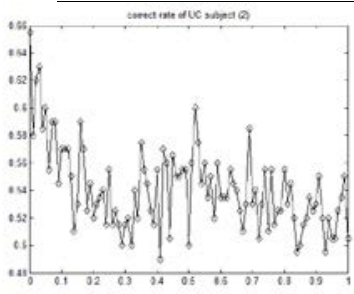

(1)

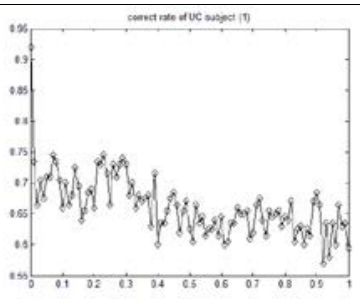

(2)

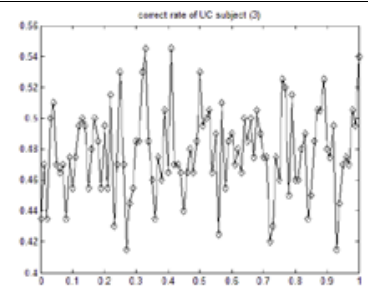

(3)

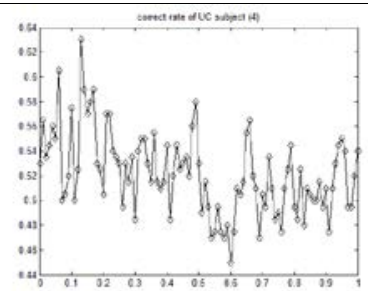

(4)

Fig. 7: The correct rate of changing UC

\section{Conclusion}

EEG characteristics is influenced by the external environment such as noise, light interference, psychological environment etc. All of these will product some extent offsets, which would make the correct classification rate decreased and affect the performance of the BCI system. This chapter focuses on the time-varying characteristics of the EEG, when use the aLDA and LDA algorithm, then regulate UC to adapt to the different situation to get better classification result.

\section{References}

[1] S.L. Xue and J. Li, The Motor Imagery EEG Processing Technology Research, pp. 36 - 47. Harbin, China: Harbin Engineering University (2013).

[2] G. Schalk, D.J. McFarland, T. Hinterberger, N. Birbaumer, and J.R. Wolpaw, a general-purpose brain- computer interface (BCI) system, pp. 1034 - 1043. IEEE Trans Biomed Eng, 51(6) (2004). 
[3] W.J. Ye and Z.J. Li, Development of a Wearable and Portable Rehabilitation Exoskeleton Robot, pp. 28 - 30. China: Journal of Integration Technology, Vol. 2 No. 4 Jul (2013).

[4] Y.Z Pan, Q.Z Goh, S.Z.S. Ge, K.P. Tee, and K.S Hong, Mind Robotic Rehabilitation Based on Motor Imagery Brain Computer Interface, pp. 162 - 170. Singapore: Social Robotics (2010).

[5] N. Sriraam, Quality-on-Demand Compression of EEG Signals for Telemedicine Applications Using Neural Network Predictors, 13 pages. Hindawi Publishing Corporation: International Journal of Telemedicine and Applications, Volume (2011), Article ID 860549.

[6] B.G. Xu, A.Q. Song and S.M. Fei, Feature Extraction and Classification of EEG in Online Brain Computer Interface, pp. 1026 - 1028. Nanjing,China: ACTA E1 ECrROMCA SIMCA, v01.39 No.5 May 2011.

[7] E.P. Zhang and H.Y. Xu, The Study of EEG Feature Extraction and Classification Based on Movement Imagination, pp. 2 - 8. Shenyang,China: Northeastern University (2010).

[8] Z.S. Li, The Design of Butterworth Lowpass Filter Based on MATLAB, pp. 49 - 52. Quanzhou, China: Information Technology, VOL.27 NO.3 Mar (2003).

[9] J.Y. Zhong, W.C Lin and W.Z Kong A CSP Component Automatic Selection Method Based on Correlation Coefficient, pp. 22 - 24. Hangzhou, China: Journal of Hangzhou Dianzi University, Vol.33 No.2 (2013).

[10] Y. Wongsawat, S. Oraintara, T. Tanaka and K.R. Rao, Lossless multichannel EEG compression, pp. 1611 - 1614 . Proceedings of the International Symposium on Circuits and Systems: ISCAS 06, (2006).

[11]J. Jiang, Research on Adaptive Brain-Computer Interface Control System, pp.19 - 41. Hunan, China: National University of Defense Technology (2011).

[12] M. Zibulevsky and Y. Zeevi, Extraction of a source from multichannel data using sparse decomposition, pp. 163 - 167. Neurocomputing 49 (2002).

[13] W. Zhou, L. Zhong and H. Zhao, Feature Attraction and Classification of Mental EEG Using Approximate Entropy, Proceedings of the IEEE, The 27th Annual Conference Engineering in Medicine and Biology Society (EMBS), pp. 5975 - 5978, Shanghai, China, September 2005.

[14] S.A. Hosseini, M.A. Khalilzadeh, M.B. Naghibi-Sistani and V. Niazmand, Higher Order Spectra Analysis of EEG Signals in Emotional Stress States, The 2nd IEEE International Conference on Information Technology and Computer Science (ITCS), Kiev, Ukraine, pp. 60 - 63, July 2010.

[15] J.M. Liu, J.Q. Li and X.H. Ye, Application of chaos analytic methods based on normal EEG, Proceedings of the IEEE, The 3rd International Conference on Computational Electromagneticsand Its Applications, pp. 426-429 (2004). 